\title{
TECNOLOGÍAS DE INTERNET PARA DISEÑAR LA RED ELÉCTRICA DEL FUTURO
}

\author{
Andrés E. Estrada \\ Universidad Nacional de Nicaragua \\ aestrada@unan.edu.ni
}

(Tipo de artículo: REFLEXIÓN. Recibido el 10/08/2011. Aprobado el 20/11/2011)

\section{RESUMEN}

Actualmente, la infraestructura de la red eléctrica se encuentra en evaluación en diferentes países: la cuestión es que es antigua y ya no responde a las exigencias y necesidades que la sociedad demanda. La próxima generación de esta red, a menudo llamada "red inteligente", deberá contar con generación de energía distribuida, más capacidad de almacenamiento, decenas de millones de fuentes de energía renovable estocásticas y el uso de las tecnologías de la comunicación, tanto para permitir la armonía entre la oferta y la demanda como para incentivar un adecuado comportamiento del consumidor. Estos desafíos buscan reducir la pérdida de energía y la huella de carbono de la red, haciéndola más "inteligente" y "más verde". En este documento se discute cómo aplicar los conceptos y las tecnologías descubiertas y aplicadas en la Internet, fruto de más de cuatro décadas de investigación, al diseño de la red eléctrica inteligente. Esto es posible debido a que ambas redes están diseñadas para satisfacer las necesidades fundamentales de información y de energía, respectivamente, a través de conexiones entre proveedores dispersos geográficamente, para consumidores igualmente dispersos geográficamente. Teniendo en cuenta éstas y otras similitudes -y también diferencias fundamentales- se proponen varias áreas específicas en las que los conceptos y las tecnologías de Internet pueden contribuir al desarrollo de la siguiente generación de la red eléctrica. También se describen algunas áreas donde se pueden mejorar las operaciones de Internet aplicando la experiencia adquirida en la red eléctrica.

\section{Palabras clave}

Internet, red eléctrica, red verde, red inteligente, energía renovable.

\section{INTERNET TECHNOLOGIES TO DESIGN THE FUTURE ELECTRICAL GRID}

\section{ABSTRACT}

Currently, the grid infrastructure is being evaluated in different countries: the point is that the grid is old and does not respond to the demands and needs of society. The next generation of this grid, often called "smart grid" must have distributed energy generation, more storage capacity, tens of millions of stochastic renewable energy sources, and the use of communication technologies, both to allow harmony between supply/demand and to encourage a proper behavior of the consumer. These challenges try to reduce energy loss and carbon footprint of the network, turning it into a more "intelligent" and "greener" grid. This document discusses how to apply the concepts and discovered-applied technologies in the Internet, which constitute the result of over four decades of research, to the design of the smart grid. This is possible because both networks are designed to satisfy the basic needs, of information and energy, through links between disperse geographically suppliers and consumers. Considering these and other similarities, -and also fundamental differences-, we propose several specific areas in which concepts and Internet technologies may contribute to the development of grid's next generation. Some areas where is possible to improve Internet operations applying the experience acquired in the grid are also described.

\section{Keywords}

Internet, grid, green grid, smart grid, renewable energy.

\section{TECHNOLOGIES D'INTERNET POUR LA CONCEPTION DE LA RÉSEAU ÉLECTRIQUE DU FUTUR}

\begin{abstract}
RÉSUMÉ
Actuellement, l'infrastructure du réseau électrique est sur évaluation en pays différents : le fait est qu'il est devenu dépassé et déjà non répond pas aux exigences et nécessités que lui demande la société, La prochaine génération de ce réseau, appelé "réseau intelligent", doit avoir génération distribué d'énergie, une capacité plus grande d'emmagasinage, dizaines de millions de sources stochastiques d'énergie renouvelable et l'usage des technologies de la communication, pour permettre l'harmonie entre l'offre et le demande et pour stimuler un comportement approprié du consommateur. Ces défis essayent de réduire les pertes d'énergie et la trace de carbone du réseau, en rendant lui plus "intelligent" et plus "vert". Dans ce document on discute sur comment appliquer les concepts et les technologies découvertes et appliqués dans l'Internet, que sont le fruit de plus de quatre décennies de recherche, à la conception du réseau électrique intelligent. Cela devient possible parce que les deux réseaux sont conçus pour satisfaire las nécessités fondamentales d'information et d'énergie, à travers de branchements entre fournisseurs et consommateurs disperses géographiquement. En considérant ces et des autres similitudes -et aussi des différences fondamentales- on propose divers champs spécifiques dans lesquelles les concepts et las technologies d'Internet peuvent contribuer au développement de la prochaine génération du réseau électrique. Aussi nous décrivons quelques champs dans lesquels on peut améliorer les opérations d'Internet en appliquant l'expérience acquis sur le réseau électrique.
\end{abstract}

Mots-clés

Internet, réseau électrique, réseau vert, réseau intelligent, énergie renouvelable. 


\section{INTRODUCCIÓN}

La red eléctrica actual es un sistema a gran escala, heterogéneo y ampliamente utilizado que, globalmente, conforma una infraestructura crítica. Desafortunadamente, este sistema es antiguo y sufre al menos de cuatro grandes problemas:

1. Una sustancial fracción de la energía utilizada para generar electricidad se desperdicia, debido a las pérdidas en la generación, la transmisión y la distribución. Por ejemplo, en 2008 en EE.UU., estos desperdicios representaron más de dos tercios de la energía generada [1].

2. Gran parte de la energía eléctrica generada se origina a partir del carbón y del gas natural, que contribuyen al cambio climático global. En EE.UU. en 2008 , el $65 \%$ de la energía utilizada para generar electricidad se originó a partir de estas dos fuentes [1].

3. Típicamente, las plantas de generación se dimensionan para carga máxima, debido a que la demanda de los actuales dispositivos terminales no es elástica y está altamente correlacionada. Esto tiene un mayor impacto sobre los costos de infraestructura, por ejemplo, en Massachusetts, el $15 \%$ de la capacidad de generación se utiliza menos de 88 horas/año [2].

4. La capacidad de almacenamiento eléctrico es muy limitada; por lo tanto, continuamente es necesario ajustar la oferta y la demanda, aunque ambas puedan cambiar dramáticamente en períodos de tiempo de unas pocas horas.

Los dos primeros problemas se pueden tratar directamente mediante generación distribuida, es decir, la generación de energía a partir de decenas de millones de fuentes de pequeña escala, en lugar de unos cientos generadores masivos [3]. Las fuentes distribuidas pueden estar localizadas cerca de los consumidores, minimizando las pérdidas de transmisión. Por otra parte, estas fuentes tienden a ser renovables -por ejemplo, la energía solar y la eólica- y tienen una huella de carbono insignificante. Por lo tanto, existe un consenso generalizado de que la siguiente generación de la red eléctrica deberá contar con generación distribuida de energía a partir de decenas de millones de fuentes de energía renovable.

Esto alterará sustancialmente la naturaleza de la red, haciéndola "más verde" pero también mucho más dinámica, porque muchas de estas fuentes serán variables en el tiempo. Además, la próxima generación probablemente será "más inteligente". En una red inteligente, los operadores pueden comunicarse con los consumidores para ajustar la oferta a la demanda y para incentivarlos a reducir su demanda cuando la red esté saturada. Esto reduce la necesidad de construir generadores con exceso de capacidad, ya que la mayor parte del tiempo estarán sin uso. También se espera que los dispositivos para manejar la carga elástica y el almacenamiento sean más comunes. Esto también alterará significativamente la naturaleza de la red. El diseño y la implementación de esta nueva generación de redes inteligentes y verdes tendrán lugar en la próxima década y creará una infraestructura de red que durará más tiempo de lo que la actual ha durado.

En este trabajo, se argumenta que los conceptos y las tecnologías descubiertas y aplicadas por la Internet fruto de más de cuatro décadas de investigaciónpueden hacer contribuciones fundamentales en la arquitectura y operación de la futura red eléctrica. Esto se debe a que ambas redes fueron diseñadas para resolver problemas similares: suplir en tiempo real las necesidades de proveedores y consumidores, distribuidos geográficamente, en cuanto a información y energía. Igualmente, se identifican algunas áreas donde será posible aplicar la amplia experiencia de la red eléctrica para mejorar el funcionamiento de la Internet.

Las contribuciones principales de este trabajo son:

- Identificar las similitudes y diferencias fundamentales entre Internet y la red eléctrica.

- Identificar las áreas donde la red eléctrica puede ser más verde e inteligente utilizando conceptos y tecnologías de Internet.

- Identificar áreas donde los principios de diseño de la red eléctrica se puedan utilizar para mejorar el funcionamiento de la Internet actual.

El resto del artículo se estructura de la siguiente forma: en la sección 2 , se presenta un estudio de los trabajos relacionados; en la sección 3 , se describe el estado actual de la red eléctrica y algunas tendencias que afectan su evolución futura; en la sección 4 , se hace una comparación entre la Internet y la red eléctrica; en la sección 5 , se identifican las áreas donde las tecnologías de Internet se pueden utilizar para reverdecer y embellecer la red eléctrica; en la sección 6 , se identifican las áreas de internet que se pueden mejorar utilizando los principios de diseño de la red eléctrica y, en la sección 7, las conclusiones.

\section{TRABAJOS RELACIONADOS}

Los términos "Green IT" y "Green Networking" se han utilizado en la literatura sobre todo para referirse a la reducción del consumo de energía eléctrica en la infraestructura de TI [4-7]. Por ejemplo, se han realizado esfuerzos para apagar los interruptores de la red cuando ésta no se utiliza [4,5]. Del mismo modo, Bolla et al [6] estudiaron cómo reducir el costo energético de un enrutador de software adaptando dinámicamente el número de núcleos activos del procesador de carga. También se presentó una propuesta para descargar servicios de los centros de datos cuando no están en uso, de tal forma que los servidores del centro de datos se puedan apagar [7]. Existe una propuesta para generar energía desde fuentes más económicas [8] que, aunque no reduce la cantidad de electricidad utilizada, sí reduce su costo. A pesar de que estos esfuerzos son encomiables, los 
servidores y centros de datos representaron sólo el 1.5\% de la electricidad utilizada en los EE.UU. en 2006 [2], que corresponde a menos del $0.5 \%$ de toda la electricidad generada [1]. Por el contrario, más de la mitad de la electricidad generada se pierde en su generación, distribución y transmisión -en 2008, en EE.UU., se perdieron dos tercios de la generación. Por lo tanto, es más importante centrarse en reducir las pérdidas en la transmisión y la distribución en lugar de pensar en reducir el consumo de energía de Internet.

Recientemente, algunos grupos de investigación se han centrado en el uso de las tecnologías de Internet para mejorar la red eléctrica. El proyecto iDEaS [9], de la Universidad de Southampton, estudia el control descentralizado y la operación y administración de las futuras redes de generación de electricidad. Los proyectos de investigación SPOTLIGHT [10] y PEIR [11], en la UCLA, se centran en el uso de sensores y teléfonos inteligentes para reducir la huella de carbono de las actividades diarias. Estos proyectos se orientan principalmente al uso de Internet como una superposición de información, más que en el uso de los conceptos y las tecnologías de Internet como una forma de evolucionar la red eléctrica.

La visión que se describe en este trabajo se alinea más estrechamente con la del proyecto Lo-Cal, en la UC Berkeley [12], que busca aplicar los principios de diseño de la Internet para diseñar la próxima generación de la red eléctrica. Sin embargo, este trabajo es el primero en exponer las similitudes y diferencias entre ambas redes y el primero en demostrar el uso de conceptos y tecnologías específicos de Internet para la próxima generación de la red eléctrica.

\section{ESTADO Y EVOLUCIÓN DE LA RED ELÉCTRICA}

La red eléctrica actual tiene un núcleo similar a una malla, que interconecta unos pocos cientos de generadores de energía eléctrica para suministrar energía a cientos o miles de redes de distribución, que suministran energía eléctrica a los consumidores industriales y domésticos [13]. Esta red sufre al menos de cuatro grandes problemas:

1. Pérdidas generalizadas en la generación, transmisión y distribución. Por ejemplo, en 2008 en los EE.UU., se perdieron más de dos tercios de la energía utilizada para generar electricidad [1]. La energía eléctrica se pierde incluso después de la generación. En EE.UU., en 1995, se desperdició alrededor del $7 \%$ de la electricidad, debido a las pérdidas en la transmisión y la distribución [14]. Del mismo modo, en 1998 en la India, las pérdidas en transmisión y distribución representaron casi el $25 \%$ de toda la electricidad generada [15].

2. La generación tradicional de energía tiende a ser intensiva en carbono. En 2008, las fuentes que no contienen carbono, como las fuentes de energía nuclear y las hidroeléctricas, produjeron menos del $30 \%$ de la energía utilizada para generar electricidad en EE.UU. [1]. La generación de electricidad es el principal consumidor de carbón en este país -con 91\%- y, por lo tanto, una causante importante de la huella de carbono [16]. Aunque países como Francia y Japón generan casi el 70\% de su energía a partir de fuentes de no-carbón, esto tiende a ser una excepción más que una regla. De acuerdo con el New York Times [17], en la China se abre cada semana una planta de energía carbonodependiente, lo suficientemente grande como para atender la demanda de todos los hogares en ciudades como Dallas o San Diego.

3. Los criterios de valoración de la red eléctrica, en su mayoría, son "tontos" y poco elásticos, incapaces de aprender del estado de la red y por tanto incapaces de reducir el consumo cuando la red está sobrecargada. Por esto, los generadores de electricidad tienen que ser construidos para carga máxima, que puede ser sustancialmente mayor que el promedio de la carga de consumo.

4. El almacenamiento de energía eléctrica es escaso. Por lo tanto, la oferta y la demanda deben coincidir todo el tiempo y los operadores deben mantener reservas de "spinning" que pueden poner en línea en algunos minutos.

Cada vez es más evidente que la red eléctrica del futuro será muy diferente de la red actual [18]. La necesidad de reducir la huella de carbono ha aumentado considerablemente la inversión en fuentes renovables de energía heterogéneas como el agua, las olas, el viento y el sol. Estas fuentes tienden a ser distribuidas e inherentemente a pequeña escala [3]. Igualmente, en un esfuerzo por reducir las pérdidas en la transmisión, la generación de energía cada vez es más distribuida, en pequeñas y numerosas fuentes de generación ubicadas cerca de las fuentes de consumo. Así, en lugar de unos cuantos generadores grandes basados en carbono, la red del futuro tendrá decenas de millones de generadores de energía distribuida basados en energía renovable.

Las fuentes de energía renovable tienden a ser muy variables: un generador eólico deja de producir energía cuando hay viento y las células solares no funcionan de noche. Sin embargo, la red actual tiene medidas inadecuadas para el almacenamiento de energía, lo que dificulta un apropiado suministro de energía a partir de fuentes renovables [19]. Las tecnologías avanzadas de almacenamiento, como los supercondensadores y las baterías basadas en nanotecnología, pueden jugar un papel cada vez más importante para limar la brecha entre la oferta y la demanda [20]. Las tecnologías de almacenamiento de energía permitirán el uso de la energía almacenada localmente, cuando la red esté sobrecargada. De hecho, la energía almacenada incluso puede alimentar de nuevo a la red.

Por lo tanto, la red eléctrica actual evolucionará a una futura red con las siguientes características: 
- Decenas de millones de fuentes de energía renovable distribuidas y variables.

- Dispositivos finales más flexibles y elásticos.

- Mucho más almacenamiento.

- Uso de las tecnologías de la comunicación, tanto para igualar la oferta y la demanda de energía como para incentivar un adecuado comportamiento del consumidor.

Esta evolución no será fácil de alcanzar ya que agregar fuentes estocásticas inherentemente para lograr un suministro confiable de electricidad no es un problema trivial. El aumento sin precedentes de las fuentes de generación de energía requerirá un control descentralizado de gran tamaño, operando simultáneamente en escalas de tiempo que van desde segundos a meses. Asegurar la estabilidad de este control es un problema abierto desafiante. Del mismo modo, introducir almacenamiento en la red incrementa dramáticamente su complejidad: gestionar eficientemente fuentes de energía en línea y distribuidas geográficamente requerirá algoritmos sofisticados y un equilibrio entre los intereses en conflicto. Finalmente, los consumidores necesitan estar informados acerca del estado actual de la red en un formato comprensible $y$ que les concedan incentivos para reducir el consumo.

La idea clave en este documento es que estos problemas son similares a los de la Internet. Por lo tanto, es posible aplicar las soluciones que utilizó Internet en sus problemas para solucionar los de la red eléctrica, con algunas modificaciones.

\section{SIMILITUDES Y DIFERENCIAS}

\subsection{Similitudes}

- Ambas redes son fundamentales para la sociedad y representan sistemas a gran escala, costosos y altamente evolucionados. La red eléctrica está diseñada para operar al 99.99\% de confiabilidad, un estándar un poco más elevado que el que utiliza Internet.

- Históricamente, surgieron de un proceso complejo que incluye una federación abajo-arriba de proveedores locales descentralizados y una infraestructura nacional arriba-abajo. Al igual que las redes LAN, los proveedores locales de electricidad prestaban inicialmente sus servicios a unas pocas decenas de clientes. Las redes eléctricas se conformaron mediante un esfuerzo por incrementar la diversidad y reducir el tiempo de inactividad. Al mismo tiempo y reconociendo el valor de la infraestructura, los gobiernos crearon las redes nacionales y las empresas de telecomunicaciones, legislando por decreto un monopolio.

- Satisfacen esencialmente demandas distribuidas, de información y de energía respectivamente, desde proveedores distribuidos. El objetivo común es adecuar la oferta para suplir la demanda.
- Generalmente, los consumidores, en ambos sistemas, no son conscientes de las complejidades subyacentes. Ellos pagan una tarifa plana o basada en el uso y a cambio esperan un servicio ininterrumpido.

- Las dos tienen una estructura en tres partes, que comprende la generación, la transmisión y la distribución. En la red eléctrica, la generación corresponde a la generación de la electricidad y en la Internet a los servidores de contenido en la nube. Para la transmisión, los generadores están interconectados a través de una capa, similar a una malla, que se asemeja a la red de transmisión a larga distancia de la red eléctrica y a los enlaces de larga distancia en el núcleo de Internet. En cuanto a la distribución, la demanda de consumo se agrega a un concentrador, que se asemeja a las compañías locales de distribución en la red eléctrica y a los ISP de dos niveles en la Internet.

- Proporcionan sus servicios a los consumidores a cambio de pago. En el caso de la red eléctrica, los servicios corresponden al consumo de electricidad en los dispositivos finales; en el caso de Internet, los servicios son una amplia gama de aplicaciones que se desplazan a través de TCP/IP.

- Ambas redes diferencian entre el núcleo y las fronteras. El núcleo de Internet está optimizado para alta capacidad $y$, con frecuencia, utiliza un formato de paquete como MPLS, que es diferente del formato IP de paquetes en la frontera. El núcleo de la red eléctrica se ha optimizado para minimizar las pérdidas de transmisión y utiliza $\mathrm{AC}$ de alto voltaje o, raramente, transmisión DC, para enviarlo a través de la red de distribución.

- Tienen incorporados diversos grados de control. Desde un control muy estricto en el núcleo, hasta un grado de heterogeneidad en la red de distribución, o un ambiente ligeramente controlado al interior de una casa o edificio.

- Soportan algún grado de almacenamiento. En la red eléctrica esto corresponde a tecnologías como las baterías, los giroscopios y las bombas de almacenamiento en las hidroeléctricas [20]. En Internet corresponde a los buffers en los routers y cachés de contenido.

- Ambas proporcionan una API simple entre la infraestructura y los usuarios, correspondiendo a los IP en Internet y a los enchufes de tres pines en la red eléctrica. Esto permite la innovación independiente.

- Requieren una gestión de recursos distribuida. Las demandas, descentralizadas geográficamente, deben ser cubiertas por fuentes de suministro geográficamente diversas.

- Ambas hacen enormes inversiones en protocolos de legado y componentes. Debido a su actual 
escala, los protocolos y API se han osificado, por lo que todo cambio hecho en ellos necesariamente tienen que ser compatible con el legado.

- Las dos codifican experiencia en forma de estándares abiertos, que permite la interconexión y la interoperabilidad. En Internet son los RFC publicados por el IETF y en la red eléctrica, los estándares los codifica la International Electrotechnical Commission [21].

- Han establecido un tenue balance entre centralización y descentralización. Los servicios públicos, al igual que los ISP, le proporcionan atención y acceso al cliente y tienen la capacidad para tomar decisiones descentralizadas. Por otro lado, los ISP Tier-1, al igual que las redes eléctricas nacionales, están centralizados y típicamente son oligopolios o monopolios.

- Los acuerdos entre los componentes centrales y las redes de acceso son complejos y, generalmente, representan la idea de un acuerdo a nivel de servicio. Por ejemplo, en la red eléctrica los estándares de interconexión para fuentes de energía distribuida están codificados en los estándares ANSI/IEEE 1547 y IEC TC8; y en Internet, los estándares de interconexión entre las "redes de usuarios" y las "redes de servicios" se rigen por los Request for Comments -RFC-, como el RFC 5865.

Estas similitudes se deben tener en cuenta para pensar en la red eléctrica como una red de distribución de contenidos, diseñada para distribuir una corriente de contenido único.

\subsection{Diferencias}

Aunque comparten muchas similitudes, estas redes difieren en algunos aspectos fundamentales. Una diferencia importante son los paquetes que admiten metadatos en forma de cabeceras. Esto le permite al paquete tener un tipo y un destino, que a la vez le permite a la Internet soportar flujos multi-producto, mientras que la red eléctrica soporta un único producto. Más importante aún, Internet permite flujos punto a punto, que son difíciles de lograr en la red eléctrica. Por ejemplo, en la red eléctrica es difícil satisfacer las demandas con granularidad fina -enviar electricidad por un cable para encender una sola bombilla-, mientras que esto es trivial en la Internet.

Un segundo conjunto de diferencias es la direccionalidad de los flujos. En Internet los flujos son bidireccionales, aunque dominan los descendentes. En cambio, en la red eléctrica casi todos los flujos son descendentes, con flujos ascendentes insignificantes aunque esto va a cambiar con la introducción de la generación distribuida. Los flujos de Internet son más inteligentes, ya que un consumidor de un flujo puede seleccionar precisamente cuánto quiere consumir y desde dónde. Por el contrario, los extremos de la red eléctrica son "tontos".
Las dos infraestructuras difieren en las escalas de tiempo involucradas en el control. El flash-crowds para contenidos en Internet, se puede responder en unos pocos segundos; mientras que el rápido incremento de la demanda en la red eléctrica no permite que esto sea fácil de cumplir: activar un reactor nuclear en línea puede llevar varios días, incluso las turbinas hidroeléctricas necesitan cerca de una hora para ponerse en línea.

La transmisión a larga distancia tiene escalas diferentes en ambas redes. En Internet, una vez que se ha instalado un enlace de fibra óptica, añadir más capacidad corresponde simplemente a añadir más longitudes de onda a los transductores ópticos en los extremos del enlace. Esto permite cubrir rápidamente los niveles de la demanda. En contraste, las líneas de transmisión eléctrica están limitadas por los conductores en cada lugar y añadir más capacidad es tremendamente costoso.

Los servicios difieren en su alcance en ambas redes. Internet soporta miles de aplicaciones, mientras que la red eléctrica probablemente continuará como una red de servicios individuales.

Aunque ambas redes proporcionan cierto grado de almacenamiento, la facilidad de añadir y gestionar ese almacenamiento es muy diferente en ambas. Los costos de memoria y almacenamiento en Internet son bajos y siguen disminuyendo cada año; mientras que el almacenamiento eléctrico es escaso y caro. Los costos de almacenamiento de electricidad no disminuyen en la misma proporción que los del almacenamiento digital.

Finalmente, la Internet y la red eléctrica se diferencian en su previsibilidad. Las demandas de la red eléctrica son altamente predecibles con base en la hora, el día, la semana, el mes y así sucesivamente. Esto les permite a los operadores de la red planificar con días o incluso semanas de antelación. Esto usualmente no es posible para los proveedores de Internet, donde incluso el tráfico del núcleo de la red puede fluctuar dramáticamente y de manera impredecible en períodos de horas.

Estos hechos diferencian fundamentalmente a las dos redes y son la base para pensar en la red eléctrica como una red de distribución de contenidos para un solo archivo. Dado que el objetivo de comparar ambas redes se centra principalmente en las similitudes, es necesario tener en mente estas diferencias, a fin de no perder de vista dicho objetivo.

\section{UNA RED ELÉCTRICA VERDE E INTELIGENTE}

Teniendo en cuenta las muchas similitudes entre ambas redes, es posible pensar que las tecnologías y los principios, que han demostrado su eficacia en la Internet, se pueden aplicar para hacer más verde e inteligente a la red eléctrica. Curiosamente, los objetivos de diseño fundamentales y secundarios de la Internet [22] parecen ser casi idénticos a los de la red 
eléctrica inteligente. Parafraseando libremente, el objetivo fundamental para ambas redes es integrar efectivamente una serie de redes existentes, administradas separadamente, en un servicio común utilizando "portales". Los objetivos comunes de diseño secundarios son: 1) tolerar la pérdida de componentes individuales, 2) soportar diferentes tipos de redes subyacentes, 3) permitir la gestión de recursos distribuidos, 4) ser rentables, 5) permitir fácilmente el acoplamiento de puntos finales, y 6) ser responsables por los recursos utilizados. Por otra parte, los problemas discutidos previamente acerca de la agregación de fuentes estocásticas, la gestión de recursos distribuidos, las múltiples escalas de tiempo de control y la incentivación al usuario, son similares a los que enfrenta la Internet.

Los investigadores de Internet, en primera instancia, han resuelto estos problemas en las últimas cuatro décadas. Por ejemplo, los avances fundamentales en el modelado de la totalidad de las fuentes estocásticas les permiten a los operadores calibrar enlaces de larga distancia con gran confianza. La Internet siempre ha implementado un control descentralizado altamente escalable: sus algoritmos de control, como la programación, el control de flujo, la gestión del tráfico y el diseño de la topología, abarcan varias órdenes de magnitud en el tiempo y se han unido para ser a la vez robustos y eficientes en la práctica. Los algoritmos distribuidos a gran escala para caching cooperativo y la colocación de recursos en redes de distribución de contenidos, han direccionado el problema de la gestión de recursos distribuidos por más de una década. Por otra parte, en el contexto de Internet existe un rico cuerpo de trabajo que estudia los incentivos por buena conducta.

Teniendo en cuenta este contexto general, a continuación se detallan algunas soluciones específicas, estudiadas en la Internet, que se pueden aplicar a la red eléctrica. Esta lista no es exhaustiva, pero indica el potencial de este enfoque. Se debe estar consciente de que la red eléctrica e Internet difieren de muchas maneras, por lo que su aplicación, aunque prometedora, no será sencilla.

\subsection{Ajustar la oferta a la demanda}

En la red eléctrica, las pérdidas en la transmisión y la distribución representan entre el 7 y el $25 \%$ de toda la electricidad generada. Por lo tanto, es importante reducir la distancia que recorre la energía eléctrica. Esto es equivalente a la necesidad de reducir la demora para atender las solicitudes de contenido en Internet. Por lo que las ideas para distribuir contenidos en las redes se pueden aplicar a la red eléctrica. Específicamente, la propuesta consiste en utilizar el caching -baterías apiladas en sub-estaciones- y ajustar la oferta y la demanda peer-to-peer con microredes [23]. En los períodos de baja demanda, las baterías apiladas pueden tener exceso de electricidad debido a que una casa o negocio utiliza energía solar o eólica. Esa energía se podría liberar cuando la demanda se incremente en un momento posterior. La demanda también podría cubrirse con ajustes peer-topeer, es decir, con autos eléctricos conectados en garajes vecinos. Arulampalam et al [24] describen un ejemplo de una red de interconexión eléctrica, necesaria para el flujo de electricidad de dos vías.

\subsection{Tomografía}

Una causa importante de pérdidas en la transmisión, especialmente en países en desarrollo, es la intervención ilegal de las líneas. En la India, por ejemplo, las "chuzadas" ilegales son la principal razón de las pérdidas en transmisión y distribución. Purkayastha [15] informa que, de 1995 a 1998, la pérdida fue de entre el 22 y el $24 \%$. Esas chuzadas generalmente se descubren utilizando reflectometría de dominio de tiempo, un procedimiento efectivo pero laborioso. En Internet, la tomografía revela la matriz de tráfico de una red mediante el monitoreo de algunos puntos de agregación. Esta técnica podría aplicarse para revelar la fina estructura de la red eléctrica, lo que facilitaría la detección de las conexiones ilegales.

\subsection{Modelar las fuentes generadoras estocásticas}

En el modelado y el análisis del tráfico de Internet existe un rico cuerpo de trabajo y se ha desarrollado una base teórica profunda para estudiar las fuentes estocásticas y sus agregados [25]. Estos modelos y análisis estocásticos son fundamentales para comprender el comportamiento de los sistemas de energía renovable, como los molinos de viento y los paneles solares, cuya producción varía en el tiempo. Específicamente, estos modelos pueden agregarse a la red eléctrica como generadores, con la diversidad suficiente como para satisfacer con alto grado de confianza la carga de base.

\subsection{Redes tolerantes al retraso}

Un desarrollo reciente en la evolución de Internet es el estudio de redes en las que los datos son transportados físicamente en mulas de datos, para proporcionar redes tolerantes al retraso. Esto es similar, en principio, al transporte de paquetes de baterías en un auto. Teniendo en cuenta que un hogar típico consume alrededor de $20 \mathrm{KWh}$ de energía y que los autos eléctricos ya tienen paquetes de baterías con capacidad similar, podría ser factible y atractivo utilizar estos autos para mover energía físicamente utilizando enrutamiento DTN [26].

\subsection{Técnicas de minería de datos}

Uno de los desarrollo reciente en Internet consiste en recoger grandes cantidades de datos y utilizar técnicas de minería de datos para extraer información útil desde ellos. Por ejemplo, cada hipervínculo en un sitio web y cada clic en un hipervínculo le informa a un motor de búsqueda acerca de las preferencias del usuario. Esto puede se puede utilizar para clasificar las páginas web y para afinar los resultados de búsqueda. De la misma manera, cada clic de un interruptor de energía también revela las preferencias del usuario. Por ejemplo, si un usuario enciende el aire acondicionado durante las horas pico, esto revela que esa es demanda inelástica. $\mathrm{Si}$ se guarda el monitoreo y se recoge el 
funcionamiento de este interruptor mediante algoritmos desarrollados para el procesamiento de señales, sería posible analizar esos procesos mediante técnicas de minería de datos.

\subsection{Compatibilidad de incentivos}

Los investigadores de Internet han estudiado durante mucho tiempo el problema de la compatibilidad de incentivos, es decir, cómo hacer que los usuarios finales se comporten de forma que sea beneficiosa para la red. Específicamente, las formulaciones de la teoría de juegos del comportamiento de usuarios, junto con los principios del diseño de mecanismos, ofrecen una visión acerca de cómo utilizar el comportamiento del usuario para satisfacer una función de bienestar social. Estos puntos de vista y los análisis son aplicables a la red eléctrica, donde se espera que los clientes reduzcan el consumo durante los periodos de sobrecarga de la red y que cooperen con sus vecinos para aliviar el tamaño de carga agregada.

\subsection{Control de sobrecarga}

La red eléctrica se sobrecarga cuando la carga excede la oferta. Esto puede suceder debido a un cortocircuito, lo cual crea una demanda infinita, o debido a altas demandas correlacionadas, por ejemplo, por el uso del aire acondicionado cuando hace calor. Los algoritmos de control de flujo proactivo y reactivo, desarrollados por Internet, pueden ser aplicables a la red eléctrica. Por ejemplo, programar por división de tiempo los aparatos de aire acondicionado en un barrio podría reducir su demanda, reduciendo al mismo tiempo la carga máxima en una subestación. El despliegue de contadores de energía inteligentes hará que este tipo de sistemas de control de sobrecarga sea posible, incluso en el corto plazo.

\subsection{Algoritmos distribuidos para control}

Internet cuenta con algoritmos de control que operan en escalas de tiempo de nanosegundos programación-, milisegundos -control de flujo-, minutos -enrutamiento-, horas -gestión de tráfico- y meses -planificación topológica. Actualmente, estos algoritmos son robustos y son fáciles de encontrar y distribuir. Este tipo de algoritmos se pueden utilizar en la red eléctrica para hacer frente a los problemas de control del ajuste de la impedancia de las fuentes milisegundos-, de la contención de fallas -segundos-, del despacho -minutos- y de la planificación de topologías -años.

\section{INTERNET APRENDE DE LA RED ELÉCTRICA}

Hasta ahora se ha analizado lo que la red eléctrica puede aprender de la Internet, pero no se trata sólo de que ésta pueda beneficiarse de los conceptos y tecnologías de Internet; de hecho, Internet tiene mucho que aprender de la red eléctrica y, aunque ese no es el enfoque principal de este trabajo, a continuación se describen algunas ideas generales.

\subsection{Fijación de precios de carga máxima}

La red eléctrica ha utilizado durante mucho tiempo precios de carga máxima, donde los consumidores pagan más por la electricidad que consumen en las horas pico. Dada la eficacia de este enfoque y al hecho de que los consumidores ya están preparados y entienden que deben reducir el consumo en esos horarios, parece que los ISP podrían reducir la capacidad de sus enlaces de red, si es necesario, utilizando un enfoque similar.

\subsection{Aislamiento de fallas}

La red eléctrica ha invertido fuertemente en la capacidad para contener fallas utilizando interruptores eléctricos, con los que aísla las fallas en pequeñas regiones dejando a la red general sin problemas. Internet podría utilizar un enfoque similar, donde los desencadenantes de tráfico u otras advertencias de peligro podrían aislar los flujos o incluso puertos enteros a través de Internet. Esto podría hacer que Internet fuera más tolerante a las fallas.

\subsection{Previsión de carga}

La red eléctrica depende de la previsión de carga exacta. Las empresas de servicios públicos utilizan los modelos climáticos para predecir por adelantado la demanda para un día, una semana e incluso para meses. Estas técnicas se podrían utilizar para prevenir la carga en la Internet.

Una vez más, esta lista no pretende ser exhaustiva, sino meramente ilustrativa de las muchas áreas de investigación que se abren para Internet desde un estudio cuidadoso a la red eléctrica.

\section{CONCLUSIONES}

A más de un siglo de su creación, la red eléctrica tendrá importantes cambios en la próxima década. Con base en un examen cuidadoso a la Internet y a la red eléctrica, en este trabajo se ha demostrado que los conceptos y las técnicas descubiertas por la primera son aplicables directamente al diseño de la próxima generación de la segunda. Por otra parte, se identificaron áreas específicas en la comunidad de Internet que se pueden trabajar con la comunidad que investiga la red eléctrica, por lo que se espera que el presente trabajo pueda iniciar un diálogo entre las dos comunidades.

\section{REFERENCIAS}

[1] Energy Information Administration of the US Department of Energy. "Annual Energy Review 2008". Report DOE/EIA-0384(2008). 2009.

[2] P. Giudice. "Our energy future and smart grid communication". [Jan. 2011].

[3] K. Alanne \& A. Saari. "Distributed energy generation and sustainable development". Renewable and Sustainable Energy Reviews, Vol. 10, No. 6, pp. 539-558, 2006.

[4] M. Gupta \& S. Singh. "Greening of the Internet". Proceedings of the 2003 conference on Applications, technologies, architectures, and protocols for computer communications, ACM SIGCOMM, Karlsruhe, Germany. Aug. 25-29, 2003.

[5] B. Heller et al. "ElasticTree: Saving energy in data center networks". 7th USENIX Symposium on Networked Systems Design and Implementation, ACM NSDI 2010. Boston, USA, Apr. 28-30, 2010. 
[6] R. Bolla et al. "Energy-aware performance optimization for next-generation green network equipment". Proceedings of the 2nd ACM SIGCOMM workshop on Programmable routers for extensible services of tomorrow ACM SIGCOMM PRESTO'09. Barcelona, Spain, Aug. 17-21, 2009.

[7] V. Valancius et al. "Greening the internet with nano data centers". Proceedings of the 5th international conference on Emerging networking experiments and technologies, ACM CoNEXT'09. Rome, Italy, Dec, 1-4, 2009.

[8] A. Qureshi et al. "Cutting the electric bill for internet-scale systems". Proceedings of the 2nd ACM SIGCOMM workshop on Programmable routers for extensible services of tomorrow ACM SIGCOMM PRESTO'09. Barcelona, Spain, Aug. 17-21, 2009.

[9] iDEaS Project. http://www.ecs.soton.ac.uk/research/ projects/618 [Mar. 2011].

[10] Spotlight project. http://nesl.ee.ucla.edu/project/show/59 [Mar. 2011].

[11] PEIR Project. http://peir.cens.ucla.edu/ [Mar. 2011].

[12] LoCal Project. http://local.cs.berkeley.edu/wiki/index. php/Main_Page [Mar. 2011].

[13] A. Von Meier. "Electric power systems: a conceptual introduction". Wiley-IEEE Press, 2006.

[14] U.S. Climate Change Technology Program. "Technology Options 2005". [Apr. 2011].

[15] P. Purkayastha. "Power Sector Policies and New Electricity Bill: from Crisis to Disaster". Economic and Political Weekly, Vol. 36, No. 25, pp. 2257-2262, 2001.

[16] A. J. Simon \& R. D. Belles. "Estimated State-Level Energy Flows in 2008". [Jan. 2011].
[17] The New York Times. "Pollution From Chinese Coal Casts a Global Shadow". June 6, 2006.

[18] E. Lightner \& S. Director. "Evolution and Progress of Smart Grid Development at the Department of Energy". DOE presentation at FERC-NARUC Smart Grid Collaborative Workshop, Washington, USA. Jul 23, 2008.

[19] B. Lee \& D. Gushee. "Electricity storage: The Achilles' heel of renewable energy". Chemical Engineering Progress, Vol. 104, No. 3, pp. 29, 2008.

[20] Electricity storage technologies. [Mar. 2011].

[21] International electrotechnical commission. [Mar. 2011].

[22] D. Clark. "The design philosophy of the DARPA Internet protocols". Proceeding Symposium proceedings on Communications architectures and protocols SIGCOMM '88, Stanford, USA. Aug. 16-18, 1988.

[23] R. Lasseter \& P. Paigi. "Microgrid: A conceptual solution". Proceeding IEEE 35th Annual Power Electronics Specialists Conference, PESC 04, Aachen, Germany. Jun. 20-25, 2004.

[24] A. Arulampalam et al. "Control of power electronic interfaces in distributed generation microgrids". International Journal of Electronics, Vol. 91, No. 9, pp. 503-523, 2004.

[25] R. Mazumdar. "Performance Modeling, Loss Networks, and Statistical Multiplexing". Morgan and Claypool, 2009.

[26] S. Jain; K. Fall \& R. Patra. "Routing in a delay tolerant network". Proceeding Proceedings of the 2004 conference on Applications, technologies, architectures, and protocols for computer communications, SIGCOMM '04, Portland, USA. Aug. 30 to Sep. 3, 2004. 